Regine Eckardt \& Qi Yu

\title{
GERMAN BLOSS-QUESTIONS AS EXTREME IGNORANCE QUESTIONS
}

\section{Abstract}

We analyze German bloss as a marker of extreme ignorance questions $Q$, basically indicating that the speaker has dismissed answers to $Q$ that she initially considered likely. We argue that bloss in questions is used in three varieties that all play on this common theme. The analysis can predict that bloss questions are not synonymous to wh-in-heaven ignorance questions, even though the two question types are similar in pragmatic content.

\section{KEYWORDS}

exhaustification; domain extension; subjective meaning; can't-find-the-value questions; particles

\section{$1 \mathrm{Nur} /$ bloss in extreme ignorance questions}

The German particles nur/bloss can be used in two senses. Apart from their meaning as focus particles (translating English only) they can also be used in extreme ignorance questions (EIQ). The question in (1) is ambiguous.

(1) Wer hat bloss Spaghetti bestellt?
a. 'Who ordered only spaghetti?'
b. 'Who on earth ordered spaghetti?'
(Focus particle reading) (EIQ reading)

While both particles exhibit the EIQ reading, there are regional preferences for bloss in the South versus nur in Northern Germany. We will use bloss in our examples. Questions in the sense (1b) convey that the speaker has tried to answer the question and found it difficult. They have been described as "urgent questions" (THURMAIR 1989, Kwon 2005) and are similar to can't-find-the-value questions 
(BAyer - Obenauer 2011) or wh-in-heaven questions (DEN Dikken - Giannakidou 2002, RAWLINS 2009, OGURO 2017). ${ }^{1}$ A more nuanced description on the data is offered in Section 3.

Polar questions as well as alternative questions with EIQ nur/bloss are unacceptable. Question (2) can only be used in the sense (2a).

(2) Gibt es hier bloss ein Krankenhaus?

gives it here BLoss a hospital

a. 'Is there only a hospital (and nothing else)?'

b. Unavailable: 'I ask with urgency: Is there a hospital?'

Similarly, (3) is unacceptable with bloss in the EIQ sense.

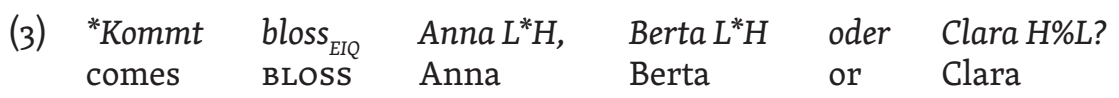

Unavailable: 'Who on earth will come: Anna, Berta or Clara?'

An analysis of EIQ nur/bloss should account not only for the EIQ reading but also for the fact that the reading is unavailable in polar questions. Given that nur/bloss in the focus particle sense can occur in assertions as well as polar questions, it would be highly stipulative to claim that they are banned from polar questions for syntactic reasons. A semantic or pragmatic explanation seems more adequate.

\section{Previous analyses}

We survey earlier analyses of EIQ in general and nur/bloss-questions in particular and argue that they all fail to explain why polar questions prohibit the use of nur/ bloss.

\subsection{Bloss-questions in German}

The first descriptive account of bloss questions was given in Thurmair $(1989,179)$ who diagnoses "reinforcement" of the question and attests that the speaker is "really interested and also poses the question to herself". She moreover shows that bloss-questions are never rhetorical and that the speaker is in search of an answer. The restriction to constituent questions does not follow from the analysis.

Kwon (2005) proposes to analyse EIQ bloss as a discourse oriented particle, as illustrated in (4). According to the analysis, EIQ bloss contributes the underlined part of the paraphrase.

\footnotetext{
1 They are not to be mixed up with Extreme Ignorance Questions in the sense of IATRIDOU - TATEVosov (2016) which convey a different speaker attitude.
} 
$\begin{array}{lllll}\text { (4) Wer hat } & \text { bloss } & & \text { Spaghetti } & \text { bestellt? } \\ \text { who has } & \text { BLOSS } & \text { spaghetti } & \text { ordered }\end{array}$ 'The only question I care for is: who ordered the coffee?'

While this analysis nicely reflects the general extension of adverbs and particles to a discourse level reading, the account fails to exclude bloss from polar and alternative questions. The potential paraphrase The only question I care for is: is there a hospital? seems a perfectly reasonable speaker attitude. Hence there is no pragmatic reason to exclude EIQ bloss from the polar question Gibt es hier ein Krankenhaus? ('Is there a hospital?'). In order to predict the prohibition, both Thurmair and Kwon have to resort to syntactic assumptions.

\subsection{Extreme ignorance questions}

DEN Dikken - Giannakidou (2002) were the first to develop an analysis of wh-on-earth questions in terms of domain widening. Using a Hamblin semantics for questions (HAMBLIN 1973), they propose that on earth modifies the wh-pronoun and extends its denotation. While the question in (5) asks for answers in a smaller search domain [[ who ]], the EIQ question in (6) takes into account a wider range of possible answers. This is, in essence, their proposal:

(5) Who ordered spaghetti?

$[[w h o]]=\{\mathbf{a} \mid \mathbf{a}$ is a person and $\mathbf{a}$ could answer the who-question $\}$

(6) Who on earth ordered spaghetti?

$[[$ who on earth $]]=\{\mathbf{a} \mid \mathbf{a}$ is a person, and $\mathbf{a}$ could answer to the who-question, possibly in an outlandish or surprising way $\}$

domain widening: $[[$ who $]] \subset[[$ who on earth $]]$

The sense of urgency or can't find the value is argued to arise by implicature from domain widening: The speaker has exhausted all reasonable answer alternatives and, being at a loss, considers more than the usual possibilities.

RAWLINS (2009) criticizes the domain widening analysis on basis of fixed domain examples like the one in (7).

(7) The jury of a game show is selecting the winner out of five candidates. A and B wait outside the jury's room for the results. The choice takes longer than usual. Speaker A wonders: Who on earth will they choose?

Rawlins argues that in the given situation, A does not consider the possibility that an unknown candidate will appear. He proposes that on earth conveys widening in the domain of possible worlds: A takes into account possible worlds that do not match 
A's earlier expectations. While the winner will be one of the five candidates it might be a winner for surprising or unexpected reasons. Rawlins develops an analysis of on earth that forces an extension of the speaker's doxastic alternatives, to include worlds that she previously considered unlikely. This extension can, but does not have to entail new individuals in [[ who ]]. The analysis is therefore consistent with observations by DEN DiKKEN - GIANNAKIDOU (2002), but generalizes their account. German bloss questions are also possible in fixed domain situations (as the next section reviews). If we assume a domain widening approach and follow Rawlins' argument, we must opt for his more general account. This, however, makes problematic predictions for polar questions. It seems perfectly rational to ask Is there a hospital? and then change one's mind and take into account worlds in which the answer is yes, there is a hospital but under unexpected circumstances. (Say, the hospital is in a chicken shed.) The case is similar to domain widening with any, as in Is there ANY hospital? A Rawlins type account hence wrongly predicts that bloss should be acceptable in polar questions. While this prediction is unproblematic for on-earth modifiers which are syntactically connected to wh-pronouns anyway, it forces us to stipulate syntactic licensing of nur/bloss by wh-pronouns without independent evidence.

\section{EIQ and dismissed answers}

\subsection{A second look at the data}

Before opting for any particular analysis, let us review the data more closely. First, we verify that German bloss-questions can be used in fixed domain situations.

(8) A and B impatiently watch a specialist trying to determine the sex of a newborn chicken. It takes very long.

A to B: Was hat das Tier bloss für ein Geschlecht?

what has the animal BLoss for a sex

'What on earth is the bird's sex?'

As in the previous example, the range of possible answers is limited - in this case to \{male, female\}. At first glance, this choice between two options is as narrow as the choice between $\{p, \neg p\}$ in polar questions. Nevertheless, bloss is perfectly possible in (8).

Second, German bloss questions are not always equivalent to domain widening questions. The question in (9) uses domain widening (KADMON - LANDMAN 1993) and invites the addressee to pose questions in the widest sense, including for example "stupid questions" or "irrelevant questions". (9) could be uttered at the end of a presentation to ask for reactions of the audience. The pragmatic effect of bloss 
in (10) is different. (10) cannot be coherently used after a presentation, or in any context where (9) is felicitous. This is indicated by \#.

(9) Haben Sie irgendwelche Fragen?

have you any questions

'Do you have any questions?'

(10) \#Was haben Sie bloss für Fragen?

what have you BLoss for questions

$\approx$ 'What the hell are your questions?"2

The speaker of (10) seems to say that she already tried to find out the addressee's questions and failed. The question also has a paradoxic ring to it, as it suggests that simply asking the addressee about her questions is not how the speaker wants to find out the answer. What is important for us, however, is that the implicatures of domain widening and those of bloss-questions must be kept apart.

Third, bloss questions are not always interchangable with German wh-on-earth questions, as the following minimal pair illustrates. Imagine a situation where a waiter wants to take orders from a guest. The guest cannot make up her mind and the waiter is getting impatient. The waiter could ask (11) (although he will definitely sound rude) but (12) is inacceptable.

(11) Was um Himmels Willen wollen Sie essen?
what in heaven's will want you eat 'What in heaven's name do you want to eat?'

(12) \#Was wollen Sie bloss essen? what want you BLoss eat

Question (12) is inadequate in this situation. It conveys that the waiter has tried to guess the answer but failed so far. The question also presupposes that there is a true answer that the waiter can find. In the given situation, however, part of the problem is that the guest has not determined the "true" answer yet.

In order to better understand the effect, consider an alternative scenario where (12) is perfectly natural. Imagine that Ada has a pet hamster that will not eat. She has offered the animal all kinds of food, but without success. Now she asks, desperately:

(13) Was willst du bloss essen?
what want you BLoss eat
'(I am at the end of my wits:) What do you want to eat?'

$2 \quad$ Native speakers may run into an exclamative interpretation for (10). This reading is not a bloss-question, as argued convincingly in THURMAIR $(1989,180)$. 
The bloss-question is adequate in contexts where the speaker has already considered at least one plausible answer and dismissed it. The waiter in (12) does not propose answers that are rejected by the guest as "false", he has to wait until the guest comes to a decision. ${ }^{3}$

Let us briefly check that bloss-questions can request an answer. This is illustrated in the following example. ${ }^{4}$

(14) Father disappeared for two hours and we were worried what happened to him. When he returns, we ask:

Wo bist $d u$ bloss gewesen?

where are you BLoss been

'Where the hell have you been?'

As in the earlier example, the speaker has considered and dismissed plausible answers to the question ('on the loo, perhaps?') but moreover, (14) urges the addressee to answer. Hence the fact that the hamster cannot talk is not decisive in example (13).

Finally, German bloss-questions cannot be used in rhetorical questions, while wh-on-earth modifiers can.

(15) Was zum Teufel hat Trump je richtig gemacht? what the devil has Trump ever right made 'What the devil has Trump ever made right?'

(16) \#Was hat Trump bloss je richtig gemacht?
what the Trump BLoss ever right made

This confirms that bloss-questions convey that the speaker does not know the answer and wants to get it. This attitude is incompatible with rhetorical questions. ${ }^{5}$

\subsection{Dismissed answers or domain widening}

The ungrammatical examples (10) and (12) show that EIQ bloss-questions differ from domain widening and wh-in-heaven questions. In both cases, the bloss-question is inappropriate in the given context because it conveys that the speaker has already considered and dismissed answers. Question (10) is unacceptable because it conveys that the speaker tried (unsuccessfully) to find out what questions the

3 For the guest it would be perfectly natural to ask (herself and/or the waiter): Was soll ich bloss essen? $=$ 'what shall I BLOss eat'. This would convey that the guest has considered and dismissed choices before.

4 A reviewer comments that they prefer bloss in questions that do not request answers (e.g., scenario (13)). Given the high frequency of bloss in information-seeking questions in our personal environment, we still consider (14) a valid data point.

5 CAPONigro - SPRouse (2007) define rhetorical questions as "question where the speaker knows the answer and believes that the addressee knows the answer". This excludes non-information-seeking questions like Wie kann man bloss so blöde sein? ('How can someone be so stupid?'). 
addressee might have, which is inappropriate in context "discussion period after a talk". In (12), the waiter conveys that she tried (unsuccessfully) to find out what the guest wants to eat. This is inappropriate in the given situation, not only because waiters do not quiz guests do you want to eat spaghetti? or do you want a steak? It is also inappropriate because the question what do you want to eat? does not have a true answer as long as the guest is still choosing: The guest determines the answer. Note the subtle difference between (12) and (7) 1-out-of-five where A and B wonder about the true answer. (7), again, would be odd if A posed the bloss question in (7) to a member of the jury.

$\begin{array}{rllll}\left(7_{\text {German }}\right) \text { A (to member of jury) } & \text { \#Wer } & \text { wird } & \text { bloss } & \text { gewinnen? } \\ & \text { who } & \text { will } & \text { BLoss } & \text { win }\end{array}$

In many cases, the speaker considers more, and unlikely answers after having dismissed plausible answers. In such cases, bloss-questions are similar to domain widening questions (we could call it a pseudo domain widening effect).

The idea of dismissing answers is challenged by fixed domain examples and in particular, examples like (8) sex of chicken with only two simple answers. In Hamblin's question semantics, the denotation of the wh-pronoun is the set of possible simple (short) answers. (8) has the simple answers \{male, female\} and dismissing one of them seems tantamount to accepting the other. This seems incompatible with the requirement that bloss-questions are still unanswered.

GroenENDIJK - StockHof's $(1982,1984)$ question semantics reveals that two simple answers $\{A, B\}$ yield four possible answers in logical space: $A$, or $B$, or $A$ and $B$, or neither $A$ nor $B$. The speaker might exclude some options, but the denotation of $Q$ is independent of the speaker's doxastic background. It is defined on $D_{s}$ which includes more worlds than only those that the speaker considers possible. $\mathrm{D}_{\mathrm{s}}$ provides worlds for all kinds of sentences and speech acts, including counterfactual worlds, fictitious worlds, worlds with monsters-worlds that can deviate from the real world in major ways. Hence there will always be worlds where answers to $Q$ are true that the speaker-with very good reasons-excludes from consideration. In the case of (8), there will be worlds where the chicken is bisexual (i.e the answer is male and female) or asexual (with an answer neither). In example (7), there are worlds where the jury decides that the prize will not be attributed this time (as recent Nobel prize committees did) or worlds where two persons win the prize together. Hence [[ Q ]] has more than the five simple answers highlighted in the scenario. Our final example, the twin case, was raised by a reviewer:

(17) Anna and Bertha Smith are twins. On their birthday, a parcel arrives sent to "Ms. Smith". No further specifications are given. Anna wonders Für wen von uns ist das bloss? ('For who of us two is the parcel bloss?'). 
While the reviewer plausibly argues that Anna and Bertha in (17) only consider the answers \{for Anna, for Bertha\}, there are in fact four possibilities: for Anna, for Bertha, for both (e.g., the sender was too greedy to spend money on two presents and decided to leave it to the twins to sort this out), and for none (the sender mixed up the address and had another Smith in mind). Of course some options are unlikely or implausible (or can be rendered implausible, for example by changing the last name from Smith to Buttigieg). But then, the domain $\mathrm{D}_{\mathrm{s}}$ includes implausible worlds as well as plausible ones. This consideration offers the basis of our analysis of EIQ bloss questions in terms of dismissed answers in the next section.

\section{The analysis}

Our analysis pursues the idea that bloss in questions conveys that answers have been dismissed. While a plain paraphrase of bloss $Q$ as "the speaker has dismissed one or more answers to Q" is too simple, we claim that the semantic components and readings of EIC bloss all play on this common theme.

We adopt Groenendijk/Stockhof's semantics of questions, notated as an equivalence relation on $\mathrm{D}_{\mathrm{s}}$. The intension of question $\mathrm{Q}$ is defined as in (18).

$$
[[\mathrm{Q}]]=\left\{\left\{\mathrm{w}:[[\mathrm{Q}]]^{\mathrm{w}}=[[\mathrm{Q}]] \mathrm{w}^{\prime}\right\}: \mathrm{w}^{\prime} \in \mathrm{D}_{\mathrm{s}}\right\}
$$

We assume that constituent questions minimally range over domains of size 2. If there is only one possible entity that can answer the wh-question, the speaker is. bound to know the answer already. Constituent questions define equivalence relations with at least four partition cells, even if the search domain in terms of HAMBLIN (1973) contains only two elements. ${ }^{6}$ Polar questions, in contrast, are characterized by the fact that the partition contains two cells only.

We assume that bloss/nur poses the following sortal restriction on its argument.

\section{(19) Sortal Restriction of bloss/nur}

EIQ bloss and nur take the intension of questions $Q$ as their argument. $[[$ bloss $]]([[\mathrm{Q}]])$ is defined only if $[[\mathrm{Q}]]$ contains at least three cells on $\mathrm{D}_{\mathrm{s}}$.

The sortal restriction in (19) does not depend on whether the speaker actually believes that $\mathrm{Q}$ has more than three answers that are compatible with the speaker's

6 This may cause problems for mathematical questions like "which of the square roots of four is greater than zero?" Mathematical statements systematically cause challenges for truth conditional semantics and it is a long standing debate in philosophy whether we should assume impossible worlds to answer these. We leave this issue unresolved.

$7 \quad$ Possible worlds may fall out of the picture because they fail to meet presuppositions of question $\mathrm{Q}$. These do not constitute a further answer to $\mathrm{Q}$. 
beliefs. In example (8), the speaker may firmly believe that the final answer will be male or female, and nothing else. The sortal restriction rests on the idea that a wh-question in principle allows the speaker to dismiss an answer and still not know the true answer to Q. It ensures that bloss is never used with the "wrong kind of Q". Remember that bloss $Q$ questions are still unanswered (see (16)). (19) hence excludes polar questions as arguments of EIQ bloss.

\subsection{Polysemous EIQ bloss}

We propose that bloss is polysemous between three related senses. All three play on the common theme of dismissing answers. For the moment we leave it open whether they could be derived from a common core and might be historically ordered. Section 4.3 shows how they are logically related.

READING 1: bloss $(Q)$ conveys that speaker A has dismissed answers that were previously plausible. Let $\operatorname{Dox}(\mathrm{A}, t)$ be doxastic alternatives of $\mathrm{A}$ at time $t$. Taking these as modal base, we moreover adopt a stereotypical ordering source $g$ (KRATZER 1991). For any two worlds $w, w^{\prime}, w<_{g} w^{\prime}$ iff $w$ is more normal/stereotypical/less surprising (according to A's beliefs) than $w^{\prime}$. The condition that A at time $t$ has dismissed an answer to $\mathrm{Q}$ can now be formalized as follows.

(20) There is a time $t-1$ before the utterance time $t$, and answers $a_{1}, \ldots, a_{n}$ to question Q such that

(i) $\exists w \in \operatorname{Dox}(\mathrm{A}, t-1)\left[\forall w^{\prime} \in \operatorname{Dox}(\mathrm{A}, t-1)\left(w^{\prime}<_{\mathrm{g}} w \rightarrow\left[a_{1} \vee \ldots \vee a_{n}\right]\left(w^{\prime}\right)\right)\right]$

(ii) $\operatorname{Dox}(\mathrm{A}, t) \vDash \neg a_{1} \wedge \ldots \wedge \neg a_{n}$

That is, the doxastic alternatives of $\mathrm{A}$ at time $t-1$ still included $a_{1}, \ldots, a_{n}$ as possibilities but since then, A has falsified these answers ( $n=1$ is possible). Note that the stereotypical ordering source g remains the same nevertheless. That is, (19) also entails that A now considers answers that are true at less stereotypical worlds, worlds that are more surprising for A. ${ }^{8}$ To summarize:

(21) [[ bloss $\left.\left._{1}(Q)\right]\right]$, uttered by A at time $t$, conveys

(i) non-at-issue: A has dismissed answers that were previously likely

(ii) speech act: A poses question $Q$

In open-domain situations, bloss is interpreted as bloss $1 \mathrm{Q}$.

8 We do not assume that the ordering source $g$ mirrors a probability distribution over worlds, as this would complicate the formalism unnecessarily. This is why: If we defined ' $w<_{\mathrm{g}} w$ ' iff $w$ is more likely than $w^{\prime \prime}$, we would have to cope with the problem that, as soon as A knows that $w$ is not the actual world (because the answer to $\mathrm{Q}$ in $w$ does not actually hold true), the probability ranking of worlds changes. We'd have to cope with a time-dependent ordering source, when A found out that some answers actually have probability zero. In terms of what is normal, these answers would still have been more normal than those that A considers now. 
READING 2: bloss $(Q)$ conveys that speaker A has preliminarily dismissed an answer $a_{1}$. At this point, we have to distinguish two ways to answer questions: find the answer or decide on the answer. Here are two examples.

(22) Find the answer

Ada has mislaid her key. She asks: Where is my key?

In order to answer the question, Ada must find out what the true location of the key is.

\section{(23) Decide the answer}

Ada studies the menu at the restaurant. She asks: What will I eat?

In order to answer the question, Ada has to decide on a dish. There is no external reality that defines the "true" answer.

Ada can ask bloss-questions in either situation. There are different ways in which answers can be preliminarily dismissed. When Ada has to decide what to eat, she has a limited set of choices on the menu. She can preliminarily dismiss a choice but later return to it. This may have to do with weighing the option against other choices, or bringing in further factors like health or environment. Deciding the answer is at work in example (7), seen from the perspective of the jury. Who wins can depend on different weighing of properties, and in redefining the weights the jury can decide the question of who is the winner? in different ways.

Finding out the truth is a different process. When Ada wants to find out the truth in (22), she preliminarily dismisses answers that seem too implausible to be pursued ("the key is in the fridge") even though she does not know for certain that the answer is false. As she becomes more desperate, she can bring in such possible answers again.

Yet another scenario is the one in example (8) sex of chicken from the point of view of the biologist who investigates the question. Imagine that the biologist first tests the animal for feature $m_{1}$. Feature $m_{1}$ normally indicates that the animal is female. So the biologist tentatively assumes that the chicken is female. Next she finds $m_{2}$, a feature that overrides $m_{1}$ and normally indicates that the animal is male. So the biologist dismisses her first assumption and now tentatively calls the chicken male. Next she tests for $m_{3}$, a feature that is found very rarely but overwrites $\left(m_{1} \wedge m_{2}\right)$. She finds $m_{3}$ and decides that the animal is yet female. And so on. As each combination of features defeasibly entails the animal's sex, the biologist adopts preliminary answers but can still rationally change her mind. (It is very plausible to imagine that she asks Was hat das Tier bloss für ein Geschlecht? as she goes along.)

To summarize, there are various ways in which speaker A can preliminarily dismiss an answer to $Q$ without thereby answering the question, even in fixeddomain situations. We will not provide formal definitions for each case but will 
focus on the case of defeasible inferences. This type will lead the way to the third possible reading of bloss $Q$.

We adopt LEwIs' (1988) analysis of defeasible inferences, using the modal base Dox (A,t) and stereotypical ordering source $g$ (KRATZER 1991).

(24) $m$ defeasibly entails answer $a_{1}$ to $Q$ iff

(i) $\operatorname{Dox}(A, t) \vDash m$

(ii) $\exists w \in \operatorname{Dox}(\mathrm{A}, t)\left[\forall w^{\prime} \in \operatorname{Dox}(\mathrm{A}, t)\left(w^{\prime}<_{\mathrm{g}} w \wedge m\left(w^{\prime}\right) \rightarrow a_{1}\left(w^{\prime}\right)\right)\right]$

As argued in Lewis (1988), this is consistent with $m \wedge r$ defeasibly entailing $\neg a_{1}$. If A sets her mind on $a_{1}$, it is thus consistent with A finding further facts $r$ that cause her to dismiss $a_{1}$ again. Lewis' analysis moreover entails that A moves further and further away from the range of those worlds that A initially considered stereotypical, unsurprising or normal (low, in terms of $g$ ). The answer may be $a_{1}$, but only true in worlds $w$ such that there are many worlds $w^{\prime}$ such that $w^{\prime}<{ }_{g} w$.

This is one of several ways to render precise that "A preliminarily dismissed answer $a$ ". There are more which we will not spell out. (25) uses the cover term to define the second possible sense of EIQ bloss.

(25) $\left[\left[\right.\right.$ bloss $\left.\left._{2}(\mathrm{Q})\right]\right]$, uttered by A at time $t$

(i) non-at-issue: A has preliminarily dismissed answer $a_{1}$ that was previously likely

(ii) speech act: A poses question $Q$

In this sense, bloss $Q$ is used in fixed-domain situations when the speaker believes that the addressee knows (or will know) the answer.

READING 3: bloss $(Q)$ conveys that speaker A believes that the answer to $Q$ is true under surprising circumstances; i.e. true in worlds that A previously did not take into consideration. In this sense, bloss $Q$ is equivalent to Rawlins' domain widening analysis for EIQ.

This-least specific-sense captures fixed-domain situations where the speaker does not believe that the addressee knows the answer. It is illustrated in $(7) /(8)$ when two observers speculate, but also the twin case (17) where neither twin knows the answer. It is not the business of addressee B to decide or dismiss the winning candidate in (7), or to determine sex in (8). Yet the interlocutors conjecture that somebody is-the expert, the jury. And if they conjecture, they will also infer that the expert, or jury, consider unlikely answers in surprising, far-from-normal possible worlds. Rawlins' domain widening account nicely generalizes over such situations and-correctly-predicts that EIQ can also be posed in a slightly wider sense, as in the twin example (17). To formalize. 
(26) $\left[\left[\right.\right.$ bloss $\left.\left._{3}(Q)\right]\right]$, uttered by A at time $t$

(i) non-at-issue: A believes that the answer a to $Q$ is only true in $w$ that are unnormal, less stereotypical than others.

(ii) speech act: A poses question $Q$

Bloss3 is used when the speaker believes that the addressee does not know or determine the answer.

Our three possible senses of EIQ bloss are related but appropriate in mutually exclusive contexts. For reasons of space, we cannot illustrate the uses comprehensively. We also leave it open whether bloss ${ }_{1} Q$ entails bloss ${ }_{2} Q$, and whether the use conditions should be predicted by logical strength and Gricean competition.

\subsection{Predictions}

The sortal restriction (19) ensures that EIQ bloss is restricted to wh-questions. In two out of three possible senses, bloss $Q$ conveys that the speaker has (tentatively) dismissed answers to $Q$ that s/he initially considered likely. This implicates that the speaker finds it difficult to find the answer and accounts for the can't-find-the -value undertones that were described in the literature.

The sense of urgency arises from the temporal dimension of bloss. All senses of bloss compare earlier and current beliefs of the speaker. This entails that the speaker continues her attempts to find an answer, which can mean that the question is urgent.

All senses of bloss entail that the speaker takes worlds into consideration that $\mathrm{s}$ /he initially considered unlikely (i.e., high in terms of the ordering source g). As argued by RAwLINS (2009), this can, but need not, mean that the speaker considers new simple answers (domain widening in the sense of DEN DIKKEN - GIANNAKIDOU 2002) or answers under unexpected circumstances (modal domain widening).

The reader assumes that the speaker uses bloss in the most specific sense. This allows us to explain why bloss questions can be unacceptable where wh-in-heaven questions are good. (10) is inappropriate because the speaker conveys that s/he has already guessed and dismissed plausible answers to the question What questions do you have?, which is in fact not the case. Similarly, (12) is inappropriate because the waiter has not guessed and dismissed plausible answers to What do you want to eat? The corresponding wh-in-heaven questions (and their German counterparts) are acceptable in the given situations, in line with Rawlins' modal domain widening analysis. Our polysemy analysis therefore allows us to understand the subtle differences between different kinds of domain-widening and extreme ignorance questions.

\subsection{How the three readings relate}

This section argues that none of the three readings can be dismissed, and aligns their ranges of application. Bloss ${ }_{1}$ are permitted whenever the speaker has definitely 
excluded one or more answer that was originally plausible (would have been a "normal" answer). Under these circumstances, bloss- $Q$ can be posed to experts, and request answers. Example (14) illustrates this case. Another natural scenario is given below.

(27) A has tried all conceivable ways to close an App on her computer.

In despair, she asks the expert:

Wie macht man bloss diese App aus?

how makes one BLoss this App off

'How on earth do you switch off this App?'

Bloss ${ }_{3}$ cannot predict (27) as it is limited to contexts where the speaker does not believe that the addressee knows the answer. Blossz includes tentative dismissal of normal answers, which is geared towards finite search spaces or decision questions.

The entry bloss ${ }_{2}$ can appear in questions where the speaker can decide rather than find the answer. No answer has been finally dismissed, but the speaker has tentatively sorted out possible answers. The addressee (if any) is not expected to answer the question-this would not make sense, given that there is no true answer yet. The final answer can be "normal" and the speaker does not convey that all plausible possibilities are false. This becomes clearest in limited domain deciding-questions like the following.

(28) A wonders who of two followers to marry.

Welchen von den beiden soll Ich bloss heiraten?
which of the two shall I BLoss marry
'Which one of these two should I marry, I wonder.'

By uttering (28), A does not convey that either one of the two candidates is unnormal or implausible. They can be equally plausible choices, and there need not be any further hidden more normal candidates to choose from: A just cannot make up her mind. Only in decision questions can answers tentatively be dismissed disregarding "normalness", and thus only in this case is bloss compatible with finally reaching a normal answer in a normal world. Matters are different when the speaker tries to find the true answer, like the expert testing for the sex of a chicken. Without aiming at a thorough investigation of these constellations, we assume that searches for true answers-where possibilities have been dismissed and revived-qualify as difficult searches. Thus, the world does not support the true answer in a normal way. For instance, in (8) the chicken may turn out to be male, but contrary to expectation several tests for 'female' also yielded positive results.

The version bloss ${ }_{3}$ is restricted to limited domain uses. Unlike bloss ${ }_{1}$ it cannot be used when asking an expert. Hence $\left(7_{\text {German }}\right)$ Wer wird bloss gewinnen, asked to a member of the jury, would be odd. Similarly, it would be odd to ask (8) Welches 
Geschlecht hat das Tier bloss? to the biologist who is ready to answer. The latter condition; expecting non-normal circumstances, is crucial. If we drop it, we would predict that just any question in German can be posed as a bloss-question as long as the speaker does not expect that the addressee knows the answer. Hence, the following should be acceptable, which it is not.

(29) I am addressing my computer-ignorant friend. I have not made any attempt to search for prices so far.

\#Was kostet bloss ein Tablet?

what costs BLoss a tablet

Intended, unavailable: 'I wonder what's the price of a tablet. I don't expect you to answer.'

We observe that bloss is not a permissible way to convey that the speaker does not expect the addressee to answer. (29) is lacking the sense of "the answer is so difficult to find that circumstances appear to be unnormal." Hence, bloss ${ }_{3}$ for questions with pre-existing answers and bloss ${ }_{2}$ for questions-to-be-decided need to be kept apart.

\section{Alternative questions}

The final section addresses the ban of bloss in alternative questions (AltQ) in (3). We follow (UEGAKI 2014) and assume that AltQ are disjunctions of polar questions. The particle bloss takes low scope, which entails the ban due to the sortal restrictions (19). The details of the analysis remain to be spelled out.

ROMERO (2015) offers the basis of a different explanation. She draws attention to the fact that alternative and polar questions cannot be embedded under surprise predicates.

(30) John was surprised who came/ *whether TOM or BILL came /*whether Tom came.

Romero explains this terms of focus selection. Her case builds on the observation by DRETSKe (1975) and ViLLALTA (2008) that surprise predicates can associate with focus. In addition, Romero assumes that wh-pronouns in questions are inherently focused whereas AltQ and polar questions are interpreted without any inherent focus features. Combining these assumptions, Romero assumes that surprise predicates only allow complements that carry a focus feature. Therefore, feature checking fails when AltQ or polar questions are embedded under surprise, hence the data in (30).

Dismissing condition (19), we could alternatively claim that EIQ bloss retains the need to check focus from its homonym, focus-sensitive bloss, to predict $(2) /(3)$. However, we see open issues for this analysis. For one, the analsis rests on specific assumptions about focus-based interpretation of wh-questions but not polar ques- 
tions, which we hesitate to submit to (ECKARDT 2007). Furthermore, while surprise is testably focus sensitive, EIQ bloss does not show any traces of focus sensitivity. Most notably, bloss can occur in questions with several answers (Wo kann ich bloss eine Zeitung kaufen? 'where can I bloss buy a newspaper?') which would be incompatible with focus sensitive bloss.

(31) Wo+bloss ${ }_{\text {Foc }}$ kann ich eine Zeitung kaufen?

$\approx$ what is the only place where I can buy a newspaper?

Our analysis, in contrast, derives $(2) /(3)$ from the sortal restriction (19) which is motivated by the core function of EIQ bloss: to convey that the speaker has dismissed some plausible answer(s) already. We maintain that this offers a maximally coherent account of EIQ with bloss and nur in German.

\section{REFERENCES}

BAyer, Josef - Obenauer, Hans-Georg. 2011. Discourse particles, clause structure, and question types. The Linguistic Review 28(4), pp. 449-491.

Biezma, Maria - Rawlins, Kyle. 2012. Responding to alternative and polar questions. Linguistics \& Philosophy 35(5), pp. 361-406.

CAPonigro, Ivano - SPRouse, Jon. 2007. Rhetorical questions as questions. In: PUig-WALDMÜLLER, Estela, ed. Proceedings of Sinn und Bedeutung 11. Barcelona: UFP, pp. 121-133 (online at http://mutis.upf.es/glif/pub/sub11/).

DEN Dikken, Marcel - Giannakidou, Anastasia. 2002. From Hell to polarity. Linguistic Inquiry 33, pp. 31-61.

DretSke, Fred. 1975. The content of knowledge. In: Freed, Bruce - MARRAS, Ausonio -MaYNARD, Patrick, eds. Forms of Representation. Amsterdam: North Holland Publishing Company, pp. 77-93.

ECKARDT, Regine. 2007. An analysis for inherent focus on wh-phrases. In: PUIG-WALdmüLLER, Estela, ed. Proceedings of Sinn und Bedeutung 11. Barcelona: UFP, pp. 209-228. (online at http://mutis.upf.es/glif/pub/sub11/)

GROENENDIJK, Jeroen - STOKHOF, Martin. 1982. Semantic analysis of wh-complements. Linguistics $\mathcal{E}$ Philosophy 5, pp. 75-233.

GroenendijK, Jeroen - STOKHOF, Martin. 1984. Studies on the Semantics of Questions and the Pragmatics of Answers. University of Amsterdam.

Hamblin, Charles L. 1973. Questions in Montague English. Foundations of Language 10, pp. 41-53. IAtridou, Sabine - TAtevosov, Sergeij. 2016. Our even. Linguistics \& Philosophy 39, pp. 295-331. Kadmon, Nirit - Landman, Fred. 1993. Any. Linguistics \& Philosophy 16(4), pp. 353-422. Kwon, Min-Jae. 2005. Modalpartikeln und Satzmodus. Ph.D. dissertation, LMU Munich. OGURO, Takeshi. 2017. The true nature of WH-the-hell phrases. Florida Linguistics Papers 4(2), pp. 113-126.

RAWLINS, Kyle. 2009. Extreme Ignorance Questions. LSA 2009. online (15. 3. 2019). 
Romero, Maribel. 2015. Surprise predicates, strong exhaustivity, and alternative questions. In: Proceedings of SALT 25, pp. 225-245.

SCHWARZSCHILD, Roger. 1999. AvoidF and other constraints on the placement of Focus. Natural Language Semantics 7, pp. 141-177.

Simons, Mandy. 2005. Dividing things up: The semantics of or and the modal or interaction. Natural Language Semantics 13, pp. 271-316.

THuRMaIR, Maria. 1989. Modalpartikeln. Tübingen: Niemeyer.

UEGAKI, Wataru. 2014. Japanese alternative questions are disjunctions of polar questions. Proceedings of SALT 24, pp. 42-62.

VILlalta, Elisabeth. 2008. Mood and gradability: An investigation of the subjunctive mood in Spanish. Linguistics \& Philosophy 31(4), pp. 467-522.

Acknowledgement: We would like to thank the audiences at SinFonIJA 2019, the ZAS Colloquium in Semantics, and Semantics \& Philosophy in Europe 11 (Warsaw), as well as Manfred Krifka, Mingya Liu, Maribel Romero and two anonymous reviewers for helpful comments and suggestions. All remaining unclarities are in our own responsibility. Research was funded by the DFG as part of the Forschungsgruppe 2111 "Questions at the Interfaces" (Konstanz), which we gratefully acknowledge.

\author{
Regine Eckardt \\ Department of Linguistics \\ University of Konstanz \\ Universitätsstr. 10 \\ 78457 Konstanz \\ Germany \\ regine.eckardt@uni-konstanz.de
}

Qi Yu

Department of Linguistics

University of Konstanz

Universitätsstr. 10

78457 Konstanz

Germany

qi.yu@uni-konstanz.de 